\title{
Cancer-associated fibroblasts enhance pancreatic cancer cell invasion by remodeling the metabolic conversion mechanism
}

\author{
TAO SHAN ${ }^{1 *}$, SHUO CHEN $^{1 *}$, XI CHEN $^{1}$, WAN RUN LIN ${ }^{2}$, WEI LI ${ }^{3}$, JIANCANG MA $^{1}$, \\ TAO WU ${ }^{1}$, XIJUAN CUI ${ }^{4}$, HONG JI ${ }^{1}$, YIMING LI $^{1}$ and YA'AN KANG $^{5}$
}

\begin{abstract}
${ }^{1}$ Department of General Surgery, The Second Affiliated Hospital of the Medical College, Xi'an Jiaotong University, Xi'an, Shaanxi 710004; ${ }^{2}$ Department of Pathology, Fudan University Shanghai Cancer Center, Shanghai 200032;

${ }^{3}$ Graduate School, Fourth Military Medical University, Xi'an, Shaanxi 710033; ${ }^{4}$ Department of General Surgery,

First Affiliated Hospital of the Medical College, Xi'an Jiaotong University, Xi'an, Shaanxi 710061, P.R. China;

${ }^{5}$ Department of Surgical Oncology, The University of Texas MD Anderson Cancer Center, Houston, TX 77030, USA
\end{abstract}

Received November 2, 2016; Accepted November 30, 2016

DOI: $10.3892 /$ or.2017.5479

\begin{abstract}
We investigated the mechanism of cancer-associated fibroblasts (CAFs) in promoting the invasion and metastasis of pancreatic cancer cells in a non-vascular manner. We verified the original generation of isolated cultured CAFs and normal fibroblasts (NFs) based on the expression of $\alpha$-SMA and vimentin, and we examined the cell glycolysis level through glucose consumption and lactate production experiments. The mRNA and protein expression of CAF glycolytic enzymes, lactate dehydrogenase and pyruvate kinase $\mathrm{m} 2$, were examined by RT-PCR and western blotting, respectively. In vitro culture first-generation pancreatic CAFs were collected and cultured together with pancreas cancer BxPc-3 and Panc-1 cells. Cell invasion and migration were assessed using a Transwell assay and scratch test, respectively. Mitochondrial activity was assessed by experimentally determining oxidative phosphorylation (OP) activity. The aerobic oxidation index of cancer cells was also examined. Succinate dehydrogenase, fumarate hydratase (FH), and monocarboxylate transporter 1 (MCT1) expression were examined using an MCT1-specific inhibitor to remove 'tumor-stromal' metabolic coupling to observe the influence of cell interstices on pancreas cancer progression. First-generation isolated cultured CAFs and NFs both grew well, and showed active proliferation. Glucose absorption and lactate production were significantly enhanced in CAFs compared with that in NFs. PCR and western blotting showed that the lactate dehydrogenase and pyruvate kinase $\mathrm{m} 2 \mathrm{mRNA}$
\end{abstract}

Correspondence to: Dr Xi Chen, Department of General Surgery, The Second Affiliated Hospital of the Medical College, Xi'an Jiaotong University, Xi'an, Shaanxi 710004, P.R. China

E-mail: chenxijiaoda@163.com

*Contributed equally

Key words: cancer-associated fibroblasts, pancreatic cancer, metabolic conversion and protein expression levels were increased in the CAFs. After indirect co-culture, OP was increased in the BxPc-3 and Panc-1 cells; correspondingly, succinate dehydrogenase, $\mathrm{FH}$ and MCT expression were increased. After the MCT1-specific inhibitor removed 'tumor-stromal' metabolic coupling, the migration and invasion abilities of the pancreatic cancer cells were decreased. Pancreatic CAFs can alter metabolism as well as communicate with and respond to cancer cell migration and invasion. This may be an important mechanism for promoting tumor progression in a non-vascular manner in the tumor microenvironment. The mechanism by which CAFs reshape metabolic transition requires further analysis.

\section{Introduction}

Pancreatic cancer is the fourth leading cause of cancer-related deaths in the US, with 46,420 estimated new cases and 39,590 estimated deaths each year (1). Even patients who undergo surgery for localized disease show a 5-year survival rate of only $20 \%$ (2). Therefore, it is important to determine the mechanism of pancreatic cancer progression and develop new treatments.

Tumor progression depends on microenvironmental interactions $(3,4)$. Cancer-associated fibroblasts (CAFs) are the most important host cells in the micro-ecological environment. They are formed from cells of different origins and can be derived from the differentiation of quiescent fibroblasts, epithelial, endothelial and mesenchymal stem cells. These cells play an important regulatory role in tumor occurrence and development (5).

Metabolic reprogramming is considered to be a hallmark of tumor cells (6). Aerobic glycolysis, known as the Warburg effect, is a characteristic metabolic feature of cancer cells (7). Recent studies have found that fibroblasts are forced into glycolysis, a phenomenon known as the 'anti-Warburg effect', since the changes in cell metabolism occur in stromal cells rather than in tumor cells (8). This change in cell metabolism may be associated with tumor progression (9). According to previous studies (10-12) in co-cultured cancer cell and fibroblast models, the mitochondrial mass in tumor cells is 
significantly increased compared to that in a separate cultured model. Since co-culture with fibroblasts can more accurately reflect the tumor microenvironment, the Warburg effect may not occur in in vitro experiments. Furthermore, treating tumor cells with lactate also significantly improves the mitochondrial mass, indicating a parasitic relationship between tumor cells and fibroblasts, with the tumor cells acting as 'parasites'. After modification, the stromal cells are forced to glycolysis, providing aerobic oxidation to the tumor cells. In the pancreatic cancer microenvironment, it remains unclear whether there is a metabolic coupling mechanism between the cancer cells and CAFs. In the present study, we extracted pancreatic CAFs and evaluated the ability of these cells to promote pancreatic cancer progression from a metabolic perspective.

\section{Materials and methods}

Materials. The glucose detection checkerboard and lactic acid checkerboard were obtained from the Jiancheng Institute of Biological Engineering (Nanjing, China). RIPA cracking liquid kits were purchased from Biyuntian Biological Co., Ltd. (Shanghai, China). Dimethyl sulfoxide was obtained from Sigma Co., Ltd. (Beijing, China). Dulbecco's modified Eagle's medium (DMEM) and fetal bovine serum (FBS) were purchased from HyClone (Logan, UT, USA). Transwell chambers were purchased from Millipore (Billerica, MA, USA). Matrigel and the One-Step RT-PCR kit were purchased from BD Biosciences (Franklin Lakes, NJ, USA). LDHA, PKM2, monocarboxylate transporter 1 (MCT1), SDH, fumarate hydratase (FH), matrix metalloprotease (MMP)-2 and MMP-9 and $\beta$-actin antibodies were purchased from Santa Cruz Biotechnology (Santa Cruz, CA, USA). The MCT1-specific blocker was from Sigma.

Cell cultures and treatments. Human pancreatic cancer cells [BxPc-3, Panc-1; obtained from the American Tissue Type Collection (ATCC; Manassas, VA, USA)] were maintained in DMEM supplemented with penicillin $(100 \mathrm{U} / \mathrm{ml})$, streptomycin $(100 \mu \mathrm{g} / \mathrm{ml}), 0.1 \mathrm{mM}$ nonessential amino acids, $0.2 \mathrm{mM}$ glutamine, $1 \mathrm{mM}$ pyruvate, and $10 \%$ heat-inactivated FBS and incubated in $5 \% \mathrm{CO}_{2}$ humidified atmosphere at $37^{\circ} \mathrm{C}$. Cells were grown to $80 \%$ confluence. In the invasion and migration experiments, the cells were cultured in DMEM without FBS.

CAF cell separation, culture and purification. Normal fibroblast (NF) and CAF cells were derived from patients with pancreatic cancer and pancreatic trauma from the Second Affiliated Hospital of Xi'an Jiaotong University. All patients were newly diagnosed and had not received any relevant treatment prior to surgery. Informed consent was obtained from all patients prior to obtaining the specimens. Fibroblast isolation was conducted as previously described (13). First, the tissue was trimmed to $1 \times 1 \times 1 \mathrm{~mm}$ and washed gently with phosphatebuffered saline (PBS) three times (5 min each). Next, the tissues were washed once with the medium and placed in fresh cell cultural medium containing $15 \%$ fetal calf serum, $2 \mathrm{mM}$ L-glutamine and $10 \%$ penicillin. The tissue was cut with a sterile scalpel blade and sections of cells were gently scraped with a blunt blade. The cells were cultured in an incubator for 3-5 days at $37^{\circ} \mathrm{C}$ and $5 \% \mathrm{CO}_{2}$. The medium was replaced once and every three days thereafter; after 14 days, the cells fully covered the Petri dish. When the cell density reached $80-90 \%$, the cells were digested with trypsin and regenerated at a rate of 1:3. The CAFs and NFs used in the experiment were the 3rd and 5 th generations of cells cultured in vitro, respectively, and showed no obvious aging phenotype.

Medium preparation of pancreatic CAFs and NFs. CAFs were added to 6-well plates at a density of $1.5 \times 10^{5} / \mathrm{ml}$ and rinsed with PBS after $24 \mathrm{~h}$. The medium was replaced with serum-free medium and cultured for $48 \mathrm{~h}$, after which the culture broth was collected and centrifuged to remove the cells and debris; the supernatant obtained was the CAF conditioned medium. These samples were stored at $4^{\circ} \mathrm{C}$. The conditioned medium of NFs was collected in the same manner.

Indirect co-culture model of CAFs and pancreatic cancer cells. The pancreatic cancer cells BxPc-3 and Panc-1 were added to Petri dishes at a density of $1.5 \times 10^{5} / \mathrm{ml}$; after $24 \mathrm{~h}$, CAF-CM was added and the cells were cultured for $48 \mathrm{~h}$. Cells in PBS or serum-free medium were used as controls. An inverted phase contrast microscope was used to observe the morphology and growth of pancreatic cancer cells in each Petri dish. Proteins were extracted from the cells.

Cell migration assay. Cell migration capability was evaluated using a scratch test. First, $10 \times 10^{5} \mathrm{BxPc}-3$ and Panc- 1 cells were seeded into $1.5 \mathrm{ml}$ media in each well of a 24 -well plate. The cells were grown to a confluent layer $(48 \mathrm{~h})$, and then, a scratch was made in each well using a pipette tip. Subsequently, the cells were washed gently with PBS, and then culture broths of CAFs and NFs were added to the respective wells. An image was captured at time point 0 . The cells were then incubated at $37^{\circ} \mathrm{C}$ in $5 \% \mathrm{CO}_{2}$ and images were acquired after $24 \mathrm{~h}$. The $24 \mathrm{~h}$ time point was chosen to decrease the potential impact of proliferation on the closing of the scratch. ImagePro Plus 5.0 from the NIH (Bethesda, MD, USA) was used to standardize the results.

Cell invasion assay. Cell invasion was examined using Transwell assays. Following incubation for $48 \mathrm{~h}, 3 \times 10^{4}$ cells were transferred to the top of the Matrigel-coated invasion chambers (BD Biosciences) in serum-free DMEM. DMEM containing $10 \%$ FBS was added to the lower chamber. After $24 \mathrm{~h}$, the non-invading cells were removed and the invading cells were fixed using $95 \%$ ethanol, stained with $0.1 \%$ crystal violet, and photographed at a magnification of x100 under an inverted phase contrast microscope (Olympus CKX31/41; Olympus, Tokyo, Japan). The experiments were repeated three times.

Glucose uptake assay. According to Fischer et al (14) the glucose uptake rate is reflected by the amount of $\left[{ }^{3} \mathrm{H}\right]-2 \mathrm{DG}$ taken up by the cells. After $24 \mathrm{~h}$ in serum-free culture, the medium was changed to low-sugar DMEM, $37 \mathrm{kBq} / \mathrm{ml}$ $\left[{ }^{3} \mathrm{H}\right]-2 \mathrm{DG}$ was added, and the cells were cultured for another $24 \mathrm{~h}$. After digestion, the small fraction of cells remaining was counted and other cells were lysed in $0.5 \mathrm{M} \mathrm{NaOH}$ for $15 \mathrm{~min}$; the same volume of $0.5 \mathrm{M}$ hydrochloric acid was added for neutralization. The dpm value in the cell lysate 
solution was examined using a microplate reader. $\left[{ }^{3} \mathrm{H}\right]-2 \mathrm{DG}$ intake by the cells was calculated as follows: Total cellular radioactivity - non-specific binding radioactivity)/24 $\mathrm{h}$.

Lactic acid detection in the cell culture medium. Cells in the 12-well plate were washed once with PBS, the medium was replaced with phenol-free red medium, and the cells were cultured for $20 \mathrm{~h}$. The supernatant was collected according to the instructions of the lactic acid detection kit. Lactic acid content was examined using a DRY-CHEM FDC3500 analyzer (Fuji, Tokyo, Japan); additionally, digested cells were counted. The result reflected the amount of lactic acid generated $/ 10^{6}$ cells.

Mitochondrial activity detection. After culturing the NF and CAF cells for $24 \mathrm{~h}$, the solution was used to culture pancreatic cancer cells for $24 \mathrm{~h}$. Cells grown in PBS or serum-free medium were used as blank controls. Fresh DMEM containing mitochondrial fluorescent probes (1:200) was added at $500 \mu \mathrm{l} /$ well and incubated for an additional $30 \mathrm{~min}$. The cells were stored and produced in a darkroom and immediately observed using an inverted fluorescence microscope.

$R T-P C R$. Total RNA was extracted from the cells using TRIzol reagent. In total, $2 \mu \mathrm{g}$ RNA was reversed-transcribed into firststrand cDNA using the RevertAid First Strand cDNA Synthesis kit (Thermo Scientific, Waltham, MA, USA). PCR primer sequences were as follows: MMP-2 forward primer, 5'-TGGTC CTGGTGCTCCTGGTG-3' and reverse primer, 5'-GCTGCCT GTCGGTGAGATTGG-3'; MMP-9 forward primer, 5'-TGGTC CTGGTGCTCCTGGTG-3' and reverse primer, 5'-GCTGCCT GTCGGTGAGATTGG-3'; LDHA forward primer, 5'-CCAAC ATGGCAGCCTTTTCC-3' and reverse primer, 5'-TCACGTT ACGCTGGACCAAA-3'; PKM2 forward primer, 5'-ATTATT TGAGGAACTCCGCCGCCT-3' and reverse primer, 5'-ATTC CGGGTCACAGCAATGATGG-3'; MCT1 forward primer, 5'-TCGGTATCTTTGGATGGAGAGG-3' and reverse primer, 5'-TGGTAACTTCATTTGGCTTCCC-3'; SDH forward primer, 5'-CGGAAGAGTGTATGGACCA-3' and reverse primer, 5'CGACGTAGTCCTTGTTGAC-3'; FH forward primer, 5'-GCGGATCCGGACATTGAGT-3' and reverse primer, 5'-CGAATTCTCACTTTGGACCCA-3'; $\beta$-actin forward primer, 5'-ATCGTGCGTGACATTAAGGAGAAG-3' and reverse primer, 5'-AGGAAGGAAGGCTGGAAGA GTG-3'. The PCR conditions were as follows: an initial reaction at $42^{\circ} \mathrm{C}$ for $1 \mathrm{~h}$ was used for cDNA synthesis, followed by denaturation at $94^{\circ} \mathrm{C}$ for $5 \mathrm{~min}$ and 22 cycles of the following reactions: $94^{\circ} \mathrm{C}$ for $30 \mathrm{sec}, 55^{\circ} \mathrm{C}$ for $30 \mathrm{sec}$ and $72^{\circ} \mathrm{C}$ for $30 \mathrm{sec}$. After the final cycle, the reaction was amplified at $72^{\circ} \mathrm{C}$ for $10 \mathrm{~min}$. The housekeeping gene $\beta$-actin was used as an internal reference.

Western blotting. In total, $5 \times 10^{5}$ cells in the logarithmic growth phase were added to $0.5 \mathrm{ml}$ of pre-chilled cell lysis buffer and incubated on ice for $30 \mathrm{~min}$. After centrifugation, the supernatant was collected and the protein contents were measured. The proteins were separated by $10 \%$ SDS-PAGE and blotted onto a nitrocellulose membrane by semi-dry transfer. Next, the membrane was immersed in Tris-buffered saline with Tween-20 containing 5\% skim milk for blocking followed by overnight incubation with the primary antibody at $4^{\circ} \mathrm{C}$. On the following day, the membrane was incubated with the secondary antibody conjugated to horseradish peroxidase at 1:2,000 dilution (Santa Cruz Biotechnology) at room temperature for $2 \mathrm{~h}$, and then an enhanced chemiluminescence kit (Amersham Pharmacia Biotech, Amersham, UK) was used for staining. The membrane was photographed and the results were analyzed.

Statistical analysis. Each experiment was repeated at least three times. The data are expressed as mean \pm SD and analyzed using the Student's t-test. $\mathrm{P}<0.05$ indicates a statistically significant difference.

\section{Results}

Preliminary identification of pancreas CAFs and NFs. After 10 days, cell morphology was observed using an inverted phase contrast microscope, and NFs and CAFs both showed a spindle shape (Fig. 1). The CAFs were spindle- or fusiform-shaped, had inconsistent sizes, showed dense growth and exhibited a disordered arrangement. For NFs, the cells showed multiple flat stellate shapes with similar cell sizes, were arranged in the same direction, and had a radially outward appearance (Fig. 1A). Transmission electron microcopy observations showed that CAFs had large cell nuclei, were evenly colored, and contained one or two nucleoli. In the cytoplasm, a large number of rough endoplasmic reticulum, mitochondria, and bundles of parallel subcapsular filaments were observed. For NFs, the cells had an irregular cell nucleus, were rich in rough endoplasmic reticulum in the cytoplasm, and had no filaments (Fig. 1B). Immunohistochemical results of CAFs showed that vimentin and $\alpha$-smooth muscle actin expression was positive. In NFs, vimentin showed positive expression, whereas $\alpha$-smooth muscle actin expression was negative (Fig. 1C and D).

CAF glycolytic metabolism. According to previous studies, CAF cells exhibit the 'Warburg effect', similarly to tumor cells; lactate is produced, secreted and absorbed by tumor cells to synthesize anabolic biomass to facilitate the rapid proliferation of tumor cells. In order to determine whether glycolysis occurs in CAFs, we examined the lactate levels of glucose uptake in the culture medium. The results demonstrated that glucose uptake and lactate production in CAFs were significantly increased compared to that in NFs (Fig. 2A and B). To further verify the change in glycolysis in CAFs, RT-PCR was conducted to detect the mRNA expression of the CAF glycolytic enzymes lactate dehydrogenase (LDHA) and pyruvate kinase $\mathrm{m} 2$ (PKM2). The results showed that LDHA and PKM2 were highly expressed in CAFs (Fig. 2C and E). The western blotting and PCR test results were consistent: LDHA and PKM2 protein expression in CAFs was higher than that in the control group (Fig. 2D and F). These results further confirmed that in vitro, glycolysis occurs in CAFs.

\section{Enhanced oxidative phosphorylation activity in pancreatic cancer cells after co-culture. In order to explore whether the capacity of aerobic oxidation is strengthened in cancer cells after co-culture, we observed the fluorescence intensity in}


$\mathbf{A}$
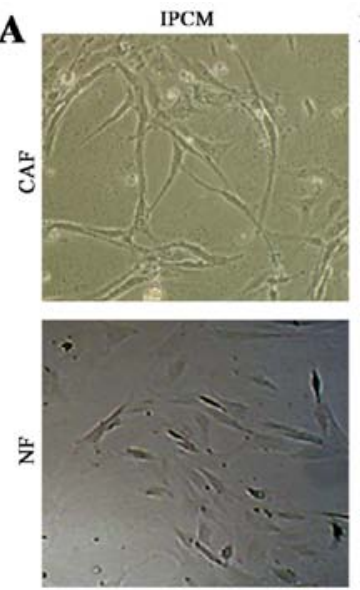
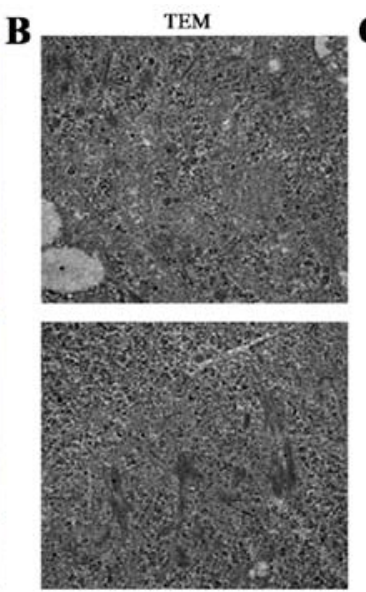
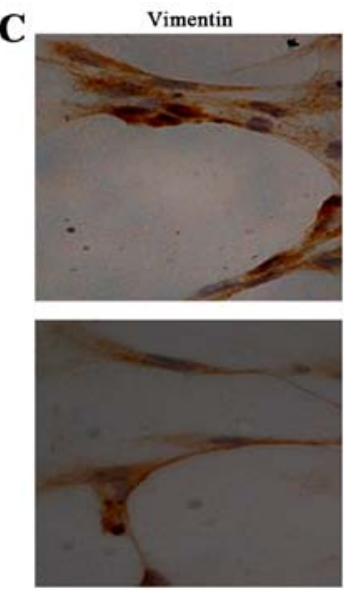
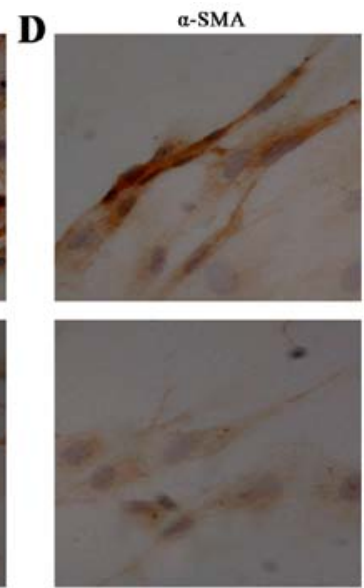

Figure 1. Preliminary identification of pancreas cancer-associated fibroblasts (CAFs) and normal fibroblasts (NFs). (A) The CAFs showed dense growth, and were disorderly arranged. For NFs, the cells were arranged in the same direction, and had a radially outward appearance. (B) TEM observations showed that CAFs had large cell nuclei, and contained one or two nucleoli. For NFs, the cells had an irregular cell nucleus. (C and D) Immunohistochemical results of CAFs showed that vimentin and $\alpha$-smooth muscle actin ( $\alpha$-SMA) expression was positive. In NFs, vimentin showed positive expression, while $\alpha$-SMA expression was negative.

$\mathbf{A}$

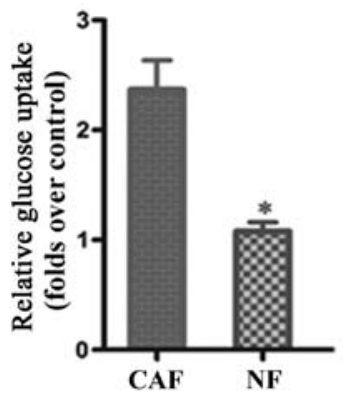

C

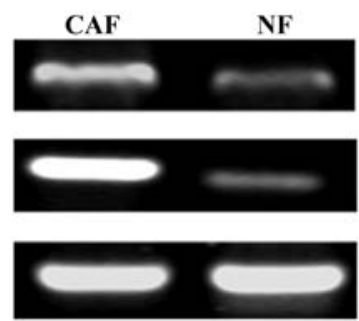

$\mathbf{E}$

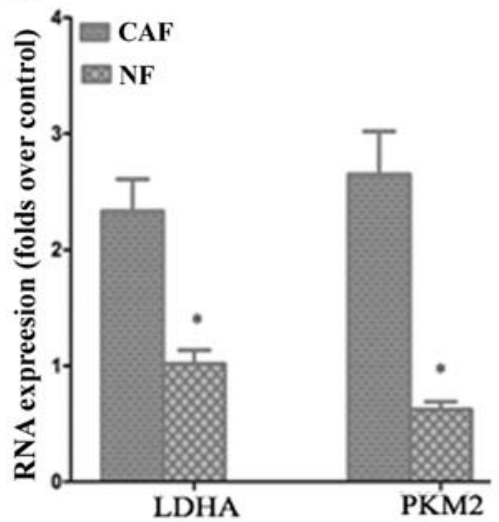

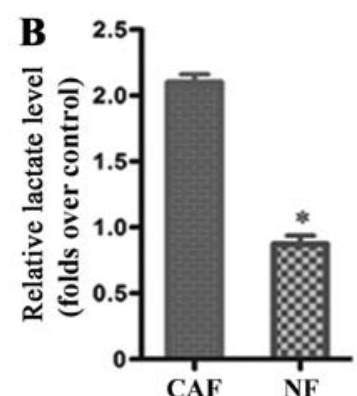

D

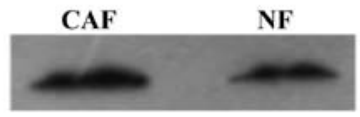

PKM2

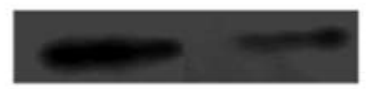

$\beta$-actin

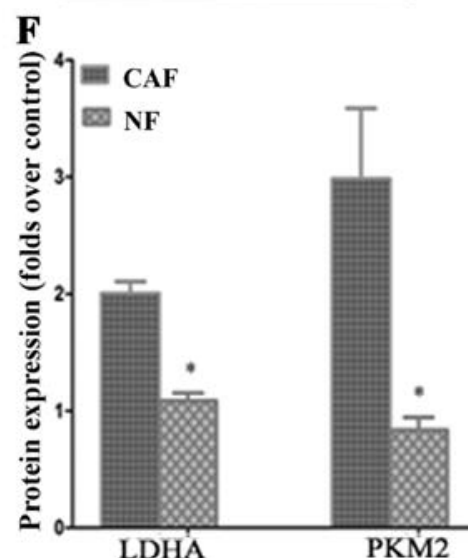

Figure 2. CAF glycolytic metabolism. (A and B) Glucose uptake and lactate production in cancer-associated fibroblasts (CAFs) were significantly increased compared to that in normal fibroblasts (NFs). (C and D) PCR and western blot results showed that LDHA and PKM2 mRNA and protien were highly expressed in CAFs. (E and F) Results of statistical analysis. The results relative to control cells are presented as a standard ratio; ${ }^{*} \mathrm{P}<0.05$ by $\mathrm{t}$-test.

the mitochondria of cancer cells by fluorescence microscopy. After co-culture, pancreatic cancer cell mitochondrial activity in the CAF group was higher than that in the NF group and cells cultured alone (Fig. 3A). The western blot results showed 


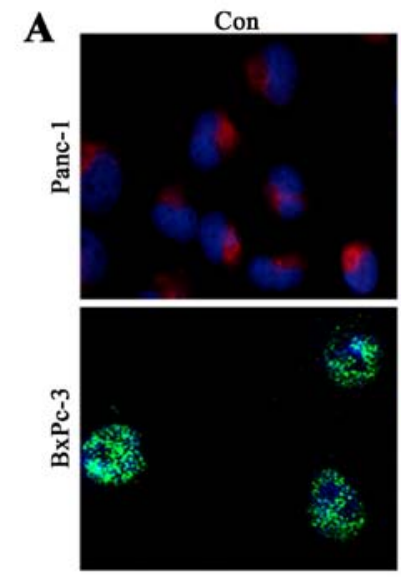

\section{$\mathbf{B}$}

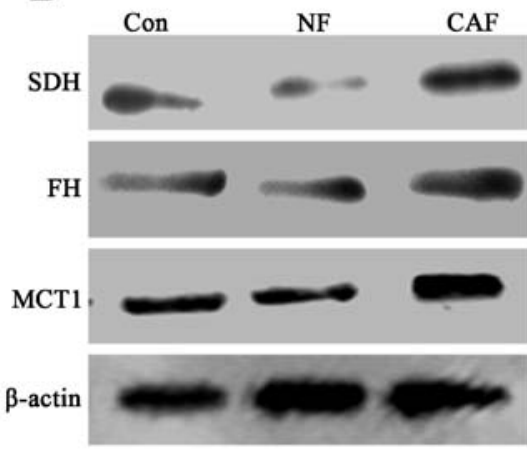

$\mathrm{NF}$

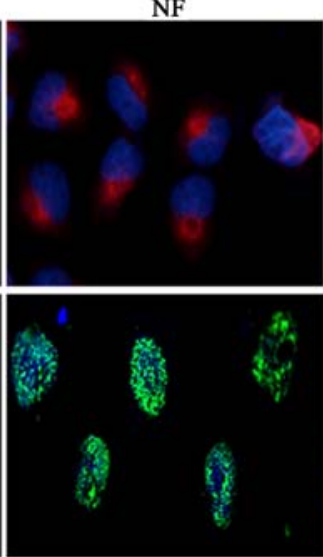

C
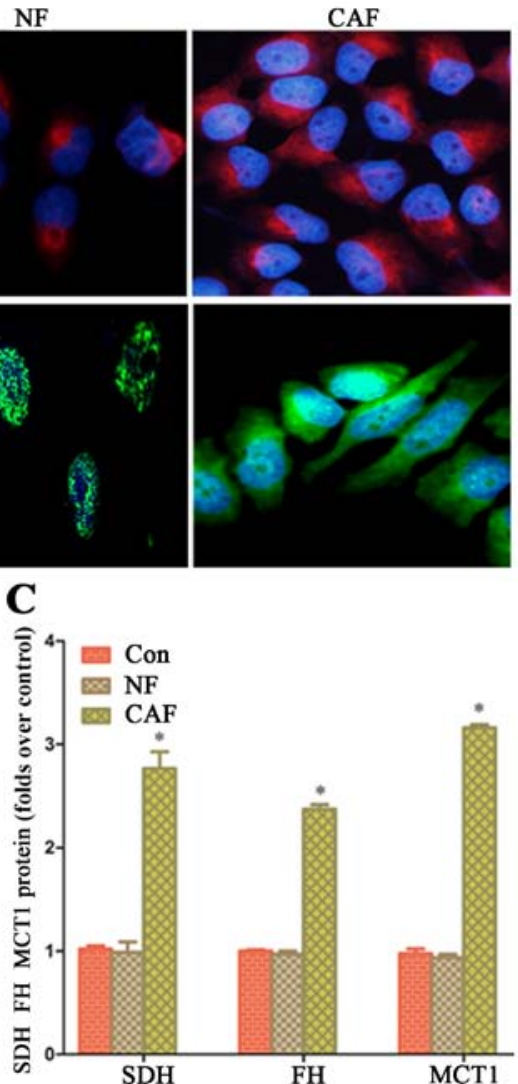

Figure 3. Enhanced oxidative phosphorylation activity in pancreatic cancer cells after co-culture. (A) After co-culture, pancreatic cancer cell mitochondria activity in cancer-associated fibroblasts (CAFs) was higher than that in normal fibroblasts (NFs) and cells cultured alone. (B and C) Western blot results showed that in cancer cells, SDH, FH and MCT1 protein expression in the CAF group was higher than that in the NF group and cells cultured alone; $\mathrm{P}<0.05$.
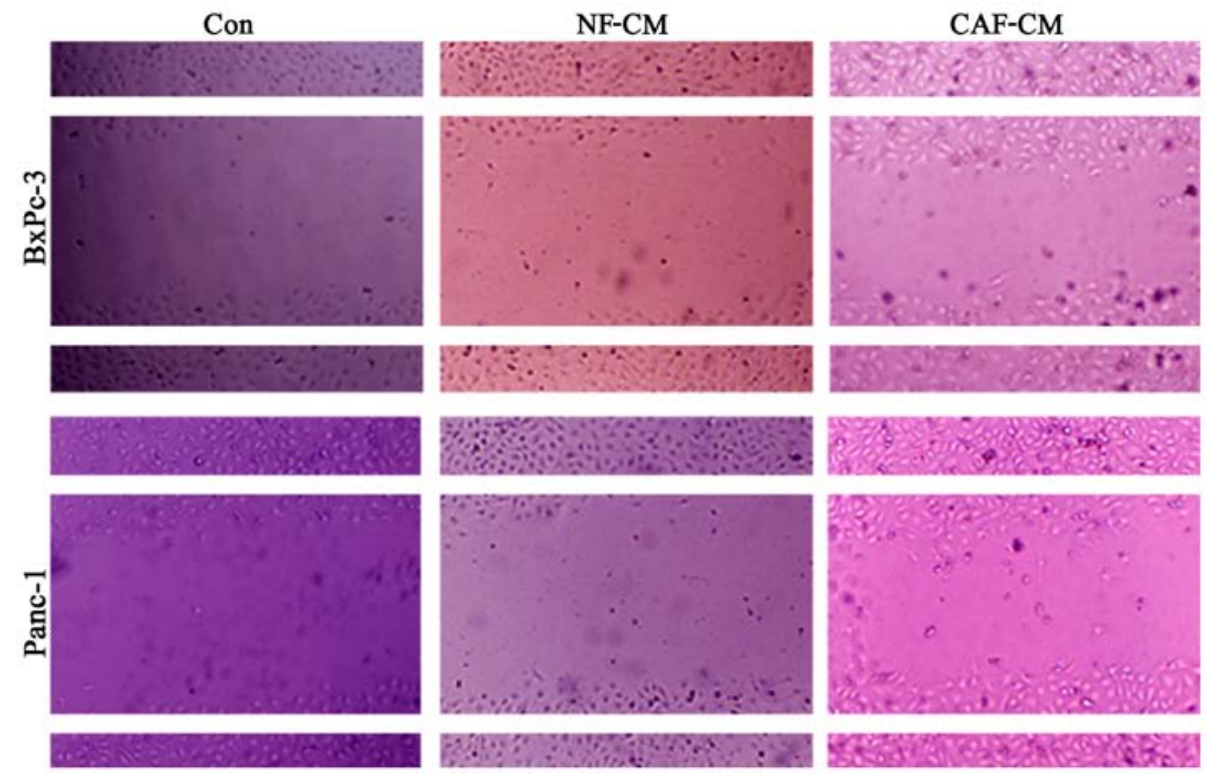

Figure 4. Cancer-associated fibroblasts (CAFs) promote the migration of pancreatic cancer cells in vitro. Scratch tests showed that compared with that in the CAF-CM group, PBS alone or NF-CM induced migration abilities in the BxPc-3 and Panc-1 cells. This indicates that CAFs enhance the migration of pancreatic cancer cells in vitro. CAF-CM, cancer-associated fibroblast-cultured medium; NF-CM, normal fibroblast-cultured medium.

that in cancer cells, SDH, FH and MCT1 protein expression in the CAF group was higher than that in the NF group and for cells cultured alone (Fig. 3B and C). This indicates that a CAF (glycolysis)-cancer cell (aerobic oxidation) metabolic coupling mode exists.
CAFs promote the migration of pancreatic cancer cells in vitro. In order to determine whether CAFs promote the migration of pancreatic cancer cells, an indirect co-culture model was developed for $48 \mathrm{~h}$, and the influence of CAFs on cancer cell migration was examined in a scratch test. The 

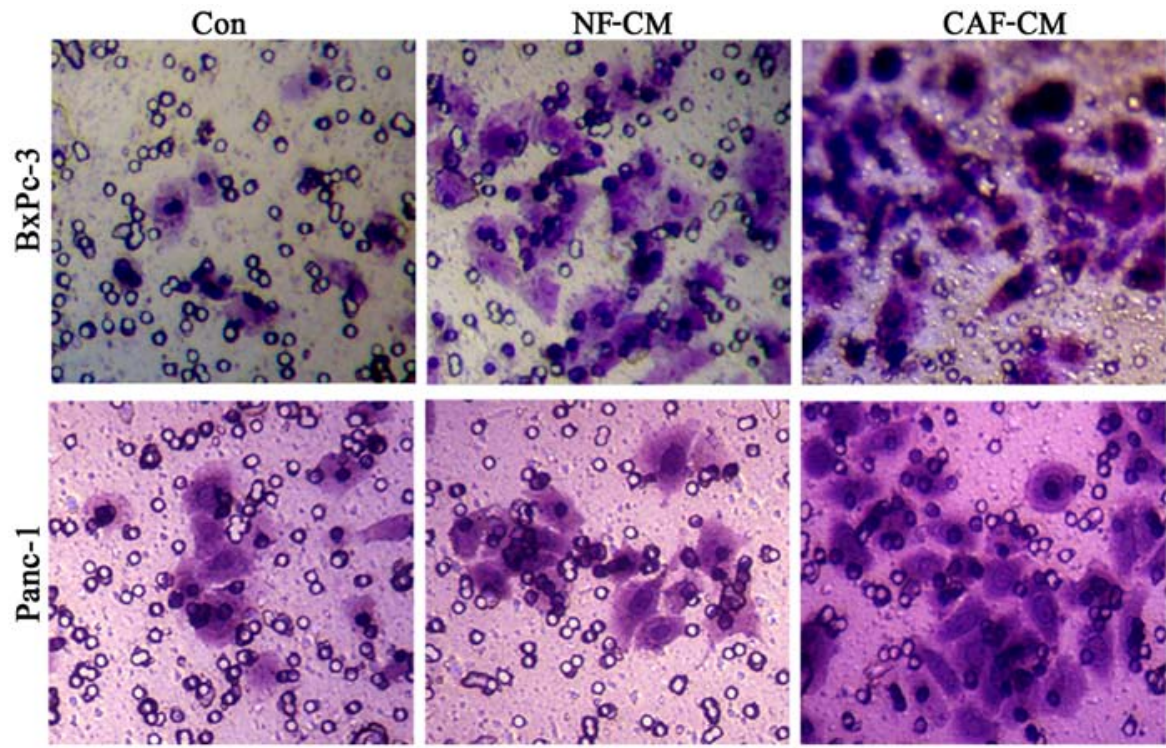

Figure 5. CAF promotes the invasion of pancreatic cancer cells in vitro. The study first adopted indirect co-culture model and process for $48 \mathrm{~h}$ and then used the Transwell assay to examine cell invasion capability. The results showed that the number of penetrated cells in the CAF-CM group was significantly increased compared to that in the PBS or NF-CM groups. CAF-CM, cancer-associated fibroblast-cultured medium; NF-CM, normal fibroblast-cultured medium.

$\mathbf{A}$
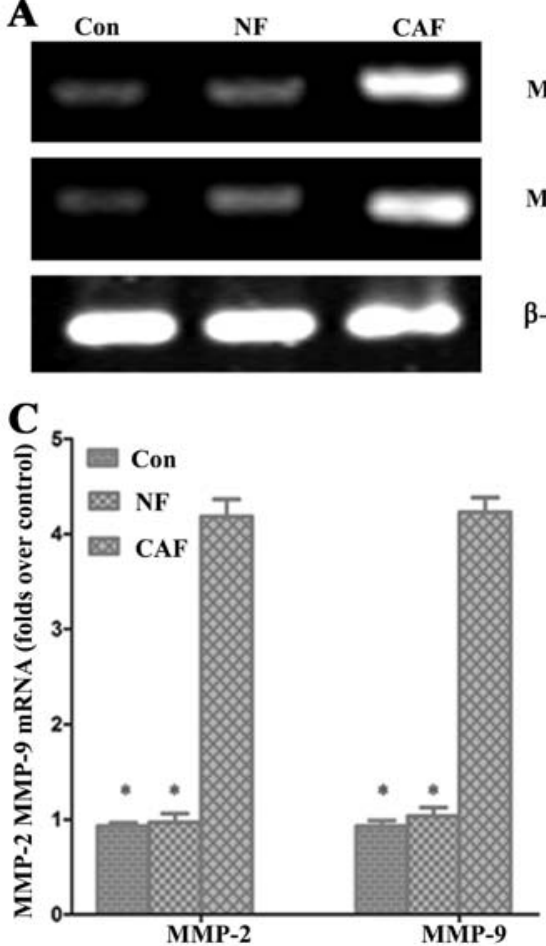

B

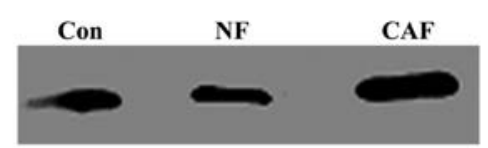

MMP-9

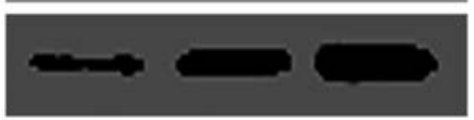

$\beta$-actin
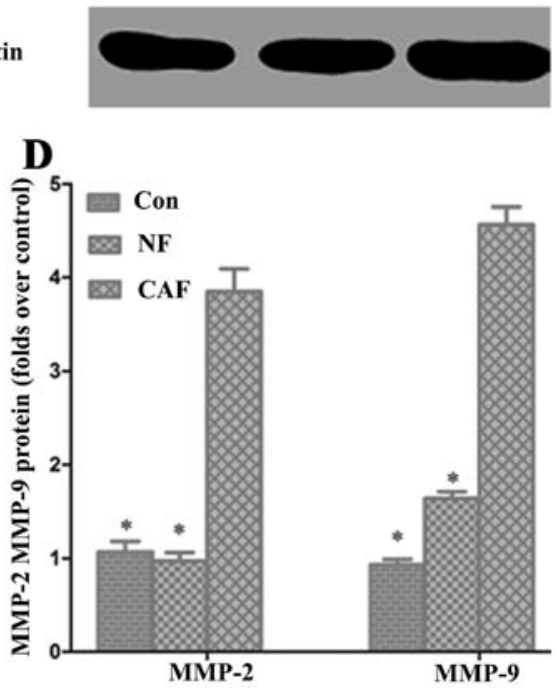

Figure 6. Effect of cancer-associated fibroblasts (CAFs) on pancreatic cancer MMP-2 and MMP-9 mRNA and protein levels. (A and C) The PCR results showed that MMP-2 and MMP-9 mRNA levels were significantly higher in the CAF-CM group than in the control group. (B and D) Western blot results showed that the levels of MMP-2 and MMP-9 protein expression were significantly increased compared to that in the control group; ${ }^{*} \mathrm{P}<0.05$. CAF-CM, cancerassociated fibroblast-cultured medium; NF-CM, normal fibroblast-cultured medium.

results showed that compared with that in the CAF-CM group, PBS alone or NF-CM induced the migration abilities in the BxPc-3 and Panc-1 cells (Fig. 4). This indicates that CAFs enhance the migration of pancreatic cancer cells in vitro.

CAF promotes the invasion of pancreatic cancer cells in vitro. In order to detect the influence of CAFs on pancreatic cancer cell invasion, we first adopted an indirect co-culture model and processed the cells for $48 \mathrm{~h}$, after which a Transwell assay was used to examine cell invasion capability. The results showed that the number of cells penetrating the CAF-CM group was significantly increased compared to that in the PBS or NF-CM groups (Fig. 5), indicating that $\mathrm{CAF}$ enhances the invasive ability of pancreatic cancer cells. 


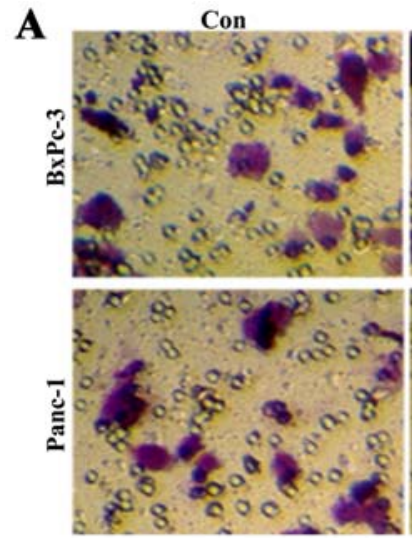

B

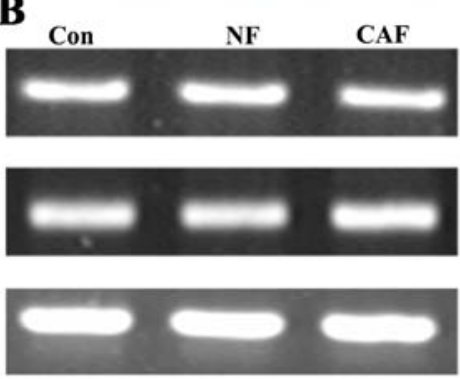

D

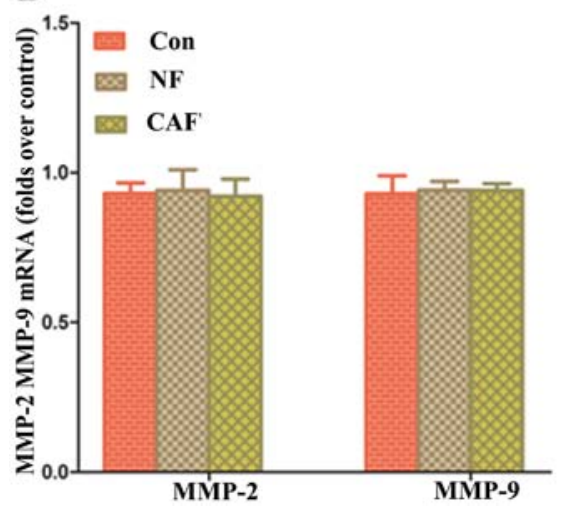

NF
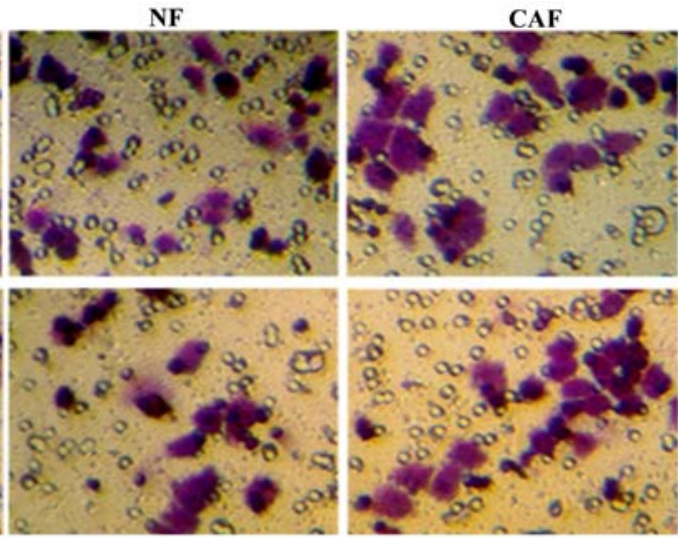

$\mathbf{C}$

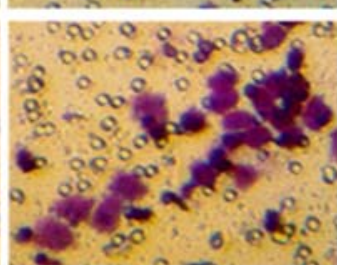

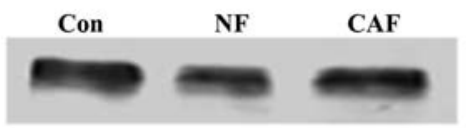

MMP-9

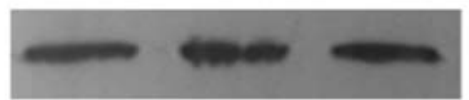

$\beta$-actin

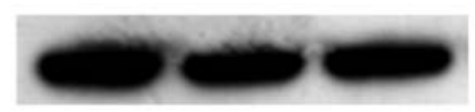

$\mathbf{E}$

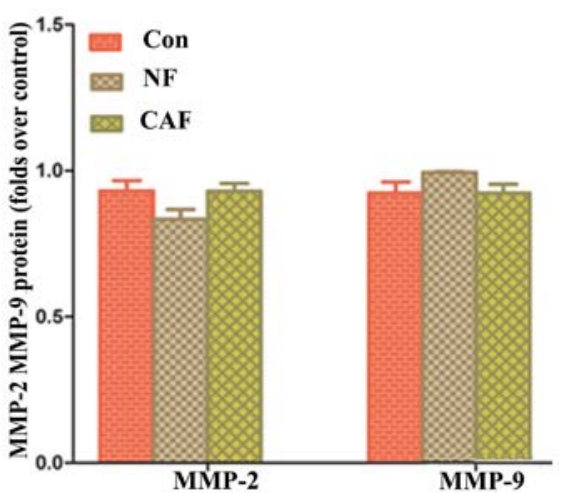

Figure 7. Impact of removing 'CAF-cancer cell' metabolic coupling upon pancreatic cancer progression. (A) The results showed that after removal of 'cancer cell-CAF' metabolic coupling, there was no significant difference in cell invasion ability among the three groups. (B and D) The PCR results showed there was no significant difference in MMP-2 and MMP-9 mRNA levels among the three groups. (C and E) The western blot results showed there was no significant difference in MMP-2 and MMP-9 protein levels among the three groups. CAF, cancer-associated fibroblast.

Effect of CAF on pancreatic cancer MMP-2 and MMP-9 $m R N A$ and protein levels. To further verify the impact of CAFs on pancreatic cancer invasion and metastasis, we used RT-PCR and western blotting to detect the expression of matrix MMP-2 and MMP-9 mRNA and protein, respectively (Fig. 6). The PCR results showed that MMP-2 and MMP-9 mRNA levels were significantly higher in the CAF-CM group than levels in the control group. Western blot results showed that the MMP-2 and MMP-9 protein expression levels were significantly increased in the CAF-CM group compared with levels in the control group (Fig. 6). These results indicate that CAF enhances cell invasion and migration abilities.

Impact of removing 'CAF-cancer cell' metabolic coupling upon pancreatic cancer progression. Based on the above results, CAFs are generally glycolytic and release lactic acid as nutrients through MCT4/1 transport; these nutrients can be used by pancreatic cancer cells to maintain their progres- sion. In order to verify whether removing metabolic coupling affects the progression of pancreatic cancer, an MCT1-specific blocker was used to pre-treat pancreatic cancer cells and for co-culture with CAFs. The results showed that after removal of 'cancer cell-CAF' metabolic coupling, there was no significant difference in the cell invasion ability among the three groups (Fig. 7). These data indicate that the 'CAF-cancer cell' metabolic coupling ring is an important mechanism for enhancing the progression of pancreatic cancer cells.

\section{Discussion}

CAFs are known to be involved in tumor progression (15). Nonetheless, the positive regulatory mechanism in tumors has not been fully elucidated. Certain growth factors are believed to be involved. Recently, several studies (16-19) examining the cell cycle described possible mechanisms from an energy metabolism perspective. Oxidative phosphorylation is considered to 
be partially abandoned in activated CAFs; thus, these cells use the glycolytic metabolic pathway instead. Through this passive metabolic pattern, released nutritional products such as lactate, can be absorbed by tumor cells to maintain growth $(8,17)$. This process is known as the reverse Warburg effect. However, whether this mechanism exists in pancreatic cancer cells was unclear. The present study revealed that pancreatic CAFs exhibit a similar reverse Warburg effect. Lactic acid secretion was significantly increased; in addition, cancer cell oxidation and metastasis were enhanced after co-culture. After blocking this coupling phenomenon, the pancreatic cancer cell migration and invasion abilities were decreased. This is consistent with results of previous studies $(11,20-22)$ indicating that changes in mesenchymal cell metabolism are important for tumor progression. Furthermore, a recently published study (23) confirmed that MCT1 and MCT4 regulated the migration and invasion of pancreatic cancer, indicating that interaction of metabolic pattern changes in the microenvironment is an important mechanism of tumor progression.

Recently, the Warburg effect has gained attention; however, previous studies have only verified that tumor cells undergo glycolysis and produce lactate $(24,25)$. However, the role of the mitochondrial respiratory chain was not determined, and the citric acid cycle and oxidative phosphorylation were thought to be reduced. Recent studies $(11,20-22)$ have revised the Warburg effect for the tumor metabolic pattern $(26,27)$. Numerous recent studies cultured only independent tumor cells in vitro, which differs from the actual in vivo tumor microenvironment, whereas the impact of the interstitial microenvironment has not been evaluated. Second, numerous studies showed that mitochondrial oxidative phosphorylation plays a positive role in tumor growth and progression. For example, in $70 \%$ of glioma patients, the mutation and inactivation of the isocitrate dehydrogenase gene was found to be associated with better prognosis and survival (28), suggesting that decreased tricarboxylic cycle activity blocks tumor progression.

In tumor cell and fibroblast co-culture models, the mitochondrial mass in the tumor cells was significantly enhanced compared with that in individual culture models (10-12). Since co-culture with CAFs more accurately reflects the microenvironment of tumorigenesis, the Warburg effects in in vitro tumor cells may not reflect the actual situation. Mitochondria mass was also significantly increased after treating tumor cells with lactate (29), indicating a parasitic relationship between tumor cells and CAFs, with the tumor cells acting as 'parasites'. After transformation, mesenchymal cells are forced to conduct glycolysis, assisting the tumor cells in conducting oxidative phosphorylation. Similarly, in co-cultured tumor cells and a CAF model, cancerous fibroblasts were induced and the expression of glycolytic metabolic enzymes was upregulated, resulting in a large number of lactate metabolites; additionally, the expression of the lactic acid transporters MCT1 and MCT4 increased accordingly in fibroblasts (30) Thus, a metabolic coupling mechanism exists between CAFs and cancer cells. We also observed 'metabolic co-existence' in pancreatic cancer studies, and the progression of pancreatic cancer was significantly affected after removing this phenomenon, again indicating a reverse Warburg effect.

In conclusion, pancreatic CAFs remodeled the metabolic transition to reflect cancer cell progression, which may be an important mechanism for promoting tumor progression in a non-vascular manner in the tumor microenvironment. Further studies may focus on the mechanism by which CAF reshapes the metabolic transition.

\section{Acknowledgements}

The present study was supported by the National Natural Science Foundation of China, NSFC (no. 81402583), the Natural Science Foundation of Shaanxi Province (no. 2014JQ4165), the Xi'an Jiaotong University Education Foundation, XJTUEF (no. xjj2014077), and the Hospital Fund of the Second Affiliated Hospital of the Health Science Center, Xi'an Jiaotong University [no. RC(XM)201402].

\section{References}

1. Siegel R, Ma J, Zou Z and Jemal A: Cancer statistics, 2014. CA Cancer J Clin 64: 9-29, 2014.

2. Winter JM, Cameron JL, Campbell KA, Arnold MA, Chang DC, Coleman J, Hodgin MB, Sauter PK, Hruban RH, Riall TS, et al: 1423 pancreaticoduodenectomies for pancreatic cancer: A single-institution experienceJ Gastrointest Surg 10: 1199-1211, 2006.

3. Koontongkaew S: The tumor microenvironment contribution to development, growth, invasion and metastasis of head and neck squamous cell carcinomas. J Cancer 4: 66-83, 2013.

4. Yoshida GJ: Metabolic reprogramming: The emerging concept and associated therapeutic strategies. J Exp Clin Cancer Res 34: $111,2015$.

5. Kalluri R and Zeisberg M: Fibroblasts in cancer. Nat Rev Cancer 6: 392-401, 2006.

6. Hanahan D and Weinberg RA: Hallmarks of cancer: The next generation. Cell 144: 646-674, 2011.

7. Warburg O: On the origin of cancer cells. Science 123: 309-314, 1956.

8. Pavlides S, Whitaker-Menezes D, Castello-Cros R, Flomenberg N, Witkiewicz AK, Frank PG, Casimiro MC, Wang C, Fortina P, Addya S, et al: The reverse Warburg effect: Aerobic glycolysis in cancer associated fibroblasts and the tumor stroma. Cell Cycle 8: 3984-4001, 2009.

9. Gonzalez CD, Alvarez S, Ropolo A, Rosenzvit C, Bagnes MF and Vaccaro MI: Autophagy, Warburg, and Warburg reverse effects in human cancer. BioMed Res Int 2014: 926729, 2014.

10. Ko YH, Lin Z, Flomenberg N, Pestell RG, Howell A, Sotgia F, Lisanti MP and Martinez-Outschoorn UE: Glutamine fuels a vicious cycle of autophagy in the tumor stroma and oxidative mitochondrial metabolism in epithelial cancer cells: Implications for preventing chemotherapy resistance. Cancer Biol Ther 12: 1085-1097, 2011.

11. Martinez-Outschoorn UE, Pavlides S, Howell A, Pestell RG, Tanowitz HB, Sotgia F and Lisanti MP: Stromal-epithelial metabolic coupling in cancer: Integrating autophagy and metabolism in the tumor microenvironment. Int J Biochem Cell Biol 43: 1045-1051, 2011.

12. Migneco G, Whitaker-Menezes D, Chiavarina B, Castello-Cros R, Pavlides S, Pestell RG, Fatatis A, Flomenberg N, Tsirigos A, Howell A, et al: Glycolytic cancer associated fibroblasts promote breast cancer tumor growth, without a measurable increase in angiogenesis: Evidence for stromal-epithelial metabolic coupling. Cell Cycle 9: 2412-2422, 2010.

13. Walter K, Omura N, Hong SM, Griffith M and Goggins M: Pancreatic cancer associated fibroblasts display normal allelotypes. Cancer Biol Ther 7: 882-888, 2008.

14. Fischer Y, Thomas J, Sevilla L, Muñoz P, Becker C, Holman G, Kozka IJ, Palacín M, Testar X, Kammermeier H, et al: Insulin-induced recruitment of glucose transporter 4 (GLUT4) and GLUT1 in isolated rat cardiac myocytes. Evidence of the existence of different intracellular GLUT4 vesicle populations. J Biol Chem 272: 7085-7092, 1997.

15. Franco OE, Shaw AK, Strand DW and Hayward SW: Cancer associated fibroblasts in cancer pathogenesis. Semin Cell Dev Biol 21: 33-39, 2010. 
16. Balliet RM, Capparelli C, Guido C, Pestell TG, MartinezOutschoorn UE, Lin Z, Whitaker-Menezes D, Chiavarina B, Pestell RG, Howell A, et al: Mitochondrial oxidative stress in cancer-associated fibroblasts drives lactate production, promoting breast cancer tumor growth: Understanding the aging and cancer connection. Cell Cycle 10: 4065-4073, 2011.

17. Bonuccelli G, Whitaker-Menezes D, Castello-Cros R, Pavlides S, Pestell RG, Fatatis A, Witkiewicz AK, Vander Heiden MG, Migneco G, Chiavarina B, et al: The reverse Warburg effect: Glycolysis inhibitors prevent the tumor promoting effects of caveolin-1 deficient cancer associated fibroblasts. Cell Cycle 9: 1960-1971, 2010

18. Martinez-Outschoorn UE, Balliet RM, Lin Z, WhitakerMenezes D, Howell A, Sotgia F and Lisanti MP: Hereditary ovarian cancer and two-compartment tumor metabolism: Epithelial loss of BRCA1 induces hydrogen peroxide production, driving oxidative stress and $\mathrm{NF \kappa B}$ activation in the tumor stroma. Cell Cycle 11: 4152-4166, 2012.

19. Pavlides S, Tsirigos A, Vera I, Flomenberg N, Frank PG Casimiro MC, Wang C, Fortina P, Addya S, Pestell RG, et al: Loss of stromal caveolin-1 leads to oxidative stress, mimics hypoxia and drives inflammation in the tumor microenvironment, conferring the 'reverse Warburg effect': A transcriptional informatics analysis with validation. Cell Cycle 9: 2201-2219, 2010.

20. Witkiewicz AK, Kline J, Queenan M, Brody JR, Tsirigos A, Bilal E, Pavlides S, Ertel A, Sotgia F and Lisanti MP: Molecular profiling of a lethal tumor microenvironment, as defined by stromal caveolin-1 status in breast cancers. Cell Cycle 10: 1794-1809, 2011.

21. Sotgia F, Martinez-Outschoorn UE, Pavlides S, Howell A, Pestell RG and Lisanti MP: Understanding the Warburg effect and the prognostic value of stromal caveolin-1 as a marker of a lethal tumor microenvironment. Breast Cancer Res 13: 213, 2011.

22. Pavlides S1, Vera I, Gandara R, Sneddon S, Pestell RG, Mercier I, Martinez-Outschoorn UE, Whitaker-Menezes D, Howell A, Sotgia F, et al: Warburg meets autophagy: Cancer-associated fibroblasts accelerate tumor growth and metastasis via oxidative stress, mitophagy, and aerobic glycolysis. Antioxid Redox Signal 16: 1264-1284, 2012.
23. Kong SC, Nøhr-Nielsen A,Zeeberg K, Reshkin SJ,Hoffmann EK, Novak I and Pedersen SF: Monocarboxylate transporters MCT1 and MCT4 regulate migration and invasion of pancreatic ductal adenocarcinoma cells. Pancreas 45: 1036-1047, 2016.

24. Koppenol WH, Bounds PL and Dang CV: Otto Warburg's contributions to current concepts of cancer metabolism. Nat Rev Cancer 11: 325-337, 2011.

25. Vander Heiden MG, Cantley LC and Thompson CB: Understanding the Warburg effect: The metabolic requirements of cell proliferation. Science 324: 1029-1033, 2009.

26. Rodríguez-Enríquez S, Gallardo-Pérez JC, Avilés-Salas A, Marín-Hernández A, Carreño-Fuentes L, Maldonado-Lagunas V and Moreno-Sánchez R: Energy metabolism transition in multicellular human tumor spheroids. J Cell Physiol 216: 189-197, 2008.

27. Shestov AA, Mancuso A, Lee SC, Guo L, Nelson DS, Roman JC, Henry PG, Leeper DB, Blair IA and Glickson JD: Bonded cumomer analysis of human melanoma metabolism monitored by $13 \mathrm{C}$ NMR spectroscopy of perfused tumor cells. J Biol Chem 291: 5157-5171, 2016.

28. Chen JR, Yao Y, Xu HZ and Qin ZY: Isocitrate dehydrogenase (IDH) $1 / 2$ mutations as prognostic markers in patients with glioblastomas. Medicine 95: e2583, 2016.

29. Van Hée VF, Pérez-Escuredo J, Cacace A, Copetti T and Sonveaux P: Lactate does not activate NF- $\mathrm{BB}$ in oxidative tumor cells. Front Pharmacol 6: 228, 2015.

30. Curry JM, Tuluc M, Whitaker-Menezes D, Ames JA, Anantharaman A, Butera A, Leiby B, Cognetti DM, Sotgia F, Lisanti MP, et al: Cancer metabolism, stemness and tumor recurrence: MCT1 and MCT4 are functional biomarkers of metabolic symbiosis in head and neck cancer. Cell Cycle 12: 1371-1384, 2013. 